\title{
NOVAS TECNOLOGIAS PARA O TRATAMENTO DA TUBERCULOSE: O QUE AS PATENTES NOS DIZEM
}

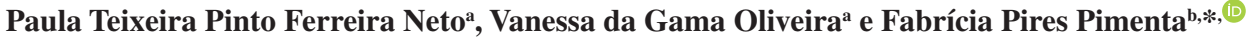 \\ ${ }^{a}$ Serviço de Farmácia, Instituto Nacional de Infectologia Evandro Chagas, 21040-360 Rio de Janeiro - RJ, Brasil \\ bNúcleo de Inovação Tecnológica, Instituto Carlos Chagas, 81350-010 Curitiba - PR, Brasil
}

Recebido em 24/04/2020; aceito em 05/05/2020; publicado na web em 30/06/2020

\begin{abstract}
NEW TECHNOLOGIES FOR THE TREATMENT OF TUBERCULOSIS: WHAT DO PATENTS TELL US. Tuberculosis still is the world's leading cause of death from a single infectious agent and has affected approximately 10 million people in 2018. The development of new technologies for treating tuberculosis is among the priority goals for the global fight against the disease. The present work makes a technological prospecting from patent documents, aiming to identify new technologies developed for the treatment of the disease, analyzing the most promising trends. The identification of new technologies was carried out through the Orbit Intelligence portal using the sentence: "(+ tuberculosis +) / TI / AB AND (A61K OR A61P) / IPC AND PRD> = 2015" as a search strategy. We identified 41 patent families with experimental evidence (in vitro / in vivo) of efficacy against Mycobacterium tuberculosis, including resistant strains. The new technologies mainly consist of chemical synthesis products. The patent protection was demanded in countries with promising markets, with potential licensors and strong intellectual property regulations. The use of patents as a source of information can contribute to orientate the development of new drugs for tuberculosis.
\end{abstract}

Keywords: tuberculosis; Mycobacterium tuberculosis; antitubercular agents; patent.

\section{INTRODUÇÃO}

A tuberculose (TB), doença infecciosa crônica causada pelo bacilo Mycobacterium tuberculosis (M. tuberculosis), continua sendo a principal causa mundial de morte ocasionada por um único agente infeccioso com cerca de 1,5 milhão de mortes em 2018. Apesar da redução do número de óbitos associados, globalmente estima-se que a doença tenha afetado cerca de 10 milhões de pessoas no ano de $2018 .{ }^{1}$

A TB é transmitida de uma pessoa infectada para uma pessoa suscetível em partículas transportadas pelo ar. Geralmente afeta os pulmões (TB pulmonar), mas pode afetar outros sítios anatômicos (TB extrapulmonar). É uma doença curável em praticamente todos os casos sensíveis aos medicamentos, desde que o tratamento seja realizado corretamente. A não adesão ao tratamento diminui a possibilidade de cura, mantém ativa a cadeia de transmissão e aumenta o risco de resistência aos medicamentos e óbitos por TB. ${ }^{2}$ Outro ponto de grande preocupação é a incidência de TB resistente a medicamentos, cujo manejo clínico requer regimes de tratamento ainda mais longos, associados a maiores riscos de eventos adversos, menor adesão e maior custo para os sistemas de saúde. ${ }^{1,3}$

Em face da persistência do problema de saúde pública em todo o mundo, a eliminação da TB está presente na Agenda 2030 para o Desenvolvimento Sustentável da Organização das Nações Unidas (ONU) entre os Objetivos de Desenvolvimento Sustentável (ODS). ${ }^{4}$ Outra iniciativa global relevante é a Estratégia END TB (pelo fim da TB) da Organização Mundial da Saúde (OMS) que propõe ousadamente reduzir as mortes por TB em $95 \%$ e reduzir novos casos em $90 \%$ até $2035 .^{5}$

Embora sejam observados esforços internacionais para deter e reverter a expansão da $\mathrm{TB}$, tais metas não serão alcançadas sem a intensificação de esforços e fomento para pesquisa e desenvolvimento $(\mathrm{P} \& \mathrm{D})$. Dentre as metas prioritárias para o enfrentamento à doença, está o desenvolvimento de novas tecnologias para TB latente, além de regimes mais simples e curtos de tratamento, inclusive para a forma resistente a medicamentos. ${ }^{1}$
No Brasil, o Programa Nacional de Controle da Tuberculose (PNCT) é responsável, entre outras ações, por estabelecer as diretrizes para o controle da doença, que tem tratamento padronizado e é exclusivamente oferecido no serviço público de saúde. O protocolo inclui esquemas terapêuticos para os diferentes perfis de resistência da doença: resistência a rifampicina (TB RR); multirresistência (TB MDR), quando há resistência a pelo menos rifampicina e isoniazida; e resistência extensiva (TB XDR), quando se identifica resistência à rifampicina e isoniazida acrescida de resistência às fluoroquinolonas e aos medicamentos de segunda linha, ou seja, aqueles já usados por resistência anterior ou por intolerância. A classificação de resistência é realizada conforme a identificação laboratorial, por meio de teste de sensibilidade fenotípico ou genotípico, das cepas de M. tuberculosis. ${ }^{2}$ Contudo, mesmo ao apresentar uma boa cobertura do tratamento, o Brasil está entre os países com alta carga da doença. ${ }^{1}$

Apesar do desenvolvimento dos novos medicamentos, delamanide e bedaquilina, aprovados há poucos anos pelas autoridades reguladoras, ${ }^{6}$ já existem relatos de resistência a esses medicamentos ${ }^{7}$ e discute-se a possibilidade da doença se tornar intratável. ${ }^{8} \mathrm{O}$ desenvolvimento de novas opções terapêuticas para combater a resistência emergente aos medicamentos é imprescindível.

No âmbito da ciência, tecnologia e inovação (CT\&I), a prospecção tecnológica tem colaborado para a identificação de oportunidades para a construção de estratégias futuras em $\mathrm{P} \& \mathrm{D}^{9}$ e os documentos de patentes têm sido considerados uma fonte excepcional de informação científica e tecnológica, na medida em que disponibilizam a informação mais recente sobre o estado da arte e, ainda, oferecem informações de caráter legal e comercial. ${ }^{10}$

O presente trabalho visa a realizar uma prospecção tecnológica a partir de documentos de patentes para identificação de novas tecnologias recém-desenvolvidas para o tratamento da TB, analisando as tendências mais promissoras em patentes. A prospecção a partir de patentes pode reduzir as incertezas da pesquisa, orientar a tomada de decisão e direcionar os investimentos em pesquisa. ${ }^{11}$ 


\section{METODOLOGIA}

A identificação de novas tecnologias desenvolvidas para o tratamento da TB foi realizada em 3 etapas. Inicialmente foi feito o levantamento de documentos de patente no portal comercial Orbit Intelligence em agosto de 2019. O portal foi selecionado por ter cobertura territorial e temporal abrangente, disponibilizando documentos de patente publicados por mais de 100 autoridades em patentes de todo o mundo, e facilidade para exportação dos resultados originados da busca. O portal Orbit Intelligence ainda permite agrupar automaticamente os documentos de patentes em famílias, o que significa dizer que são reunidos um ou mais documentos de patentes individuais relacionados a uma única invenção, ou seja, a uma única tecnologia.

Para definição dos termos a serem utilizados na busca foram consideradas as seguintes sinonímias para TB: Tuberculose, Tuberculosis, Mycobacterium tuberculosis, antituberculosos, tuberculostáticos e seus correlatos em inglês. O termo "+tuberculost" foi selecionado para construção da estratégia de busca e buscado nos campos de título (TI) ou resumo (AB).

A Classificação Internacional de Patentes (IPC) também foi utilizada na estratégia de busca. A IPC é um sistema de classificação internacional cujas áreas tecnológicas são divididas em classes, subclasses, grupos principais e grupos, através de um sistema hierárquico. Uma vez identificados os grupos aos quais o pedido de patente se refere, é possível identificar outros pedidos de patentes relacionados ao mesmo fim. Na estratégia de busca foram incluídos os códigos A61K (Cosméticos ou preparações similares para higiene pessoal) ou A61P (atividade terapêutica específica de compostos químicos ou preparações medicinais) a fim de delimitar as buscas para medicamentos.

Com intuito de identificar as mais recentes tendências em pesquisa, desenvolvimento e inovação (PD\&I), tais como tecnologias emergentes, principais desenvolvedores de novas tecnologias e países onde os depósitos ocorrem, apenas documentos com data de prioridade a partir de 2015 foram considerados para esta análise.

A estratégia de busca utilizada pode ser observada a partir da seguinte sentença: “(+tuberculos+)/TI/AB AND (A61K OR A61P)/ IPC AND PRD >= 2015”.

A segunda etapa da busca consistiu em uma análise criteriosa do conteúdo de documentos de patentes levantados a fim de incluir no presente estudo somente famílias de patentes relevantes para a temática. Adotou-se como critérios de exclusão a falta de informações formais ou técnicas, o patenteamento de tecnologias de prevenção e/ou diagnóstico, bem como as de uso veterinário, visando obter um corpus de estudo contendo somente documentos de patente de medicamentos voltadas para o tratamento da TB em humanos.

Em seguida, deu-se início a terceira etapa de seleção das tecnologias identificadas como relevantes, elegendo-se as que possuíam evidências experimentais de eficácia in vitro, especificamente para M. tuberculosis, incluindo cepas resistentes, e testes in vivo. O fluxo utilizado está ilustrado na Figura 1.

\section{RESULTADOS E DISCUSSÃO}

\section{Panorama geral}

Conforme ilustrado na Figura 1, a partir da realização da busca, descrita na primeira etapa metodológica, foram identificadas $1196 \mathrm{fa}$ mílias de documentos de patente. Após a aplicação dos critérios de exclusão da segunda etapa metodológica, foram descartadas $428 \mathrm{fa}-$ mílias. Em seguida, após a aplicação da terceira etapa metodológica, foram excluídas 481 famílias de patente por ausência de evidência

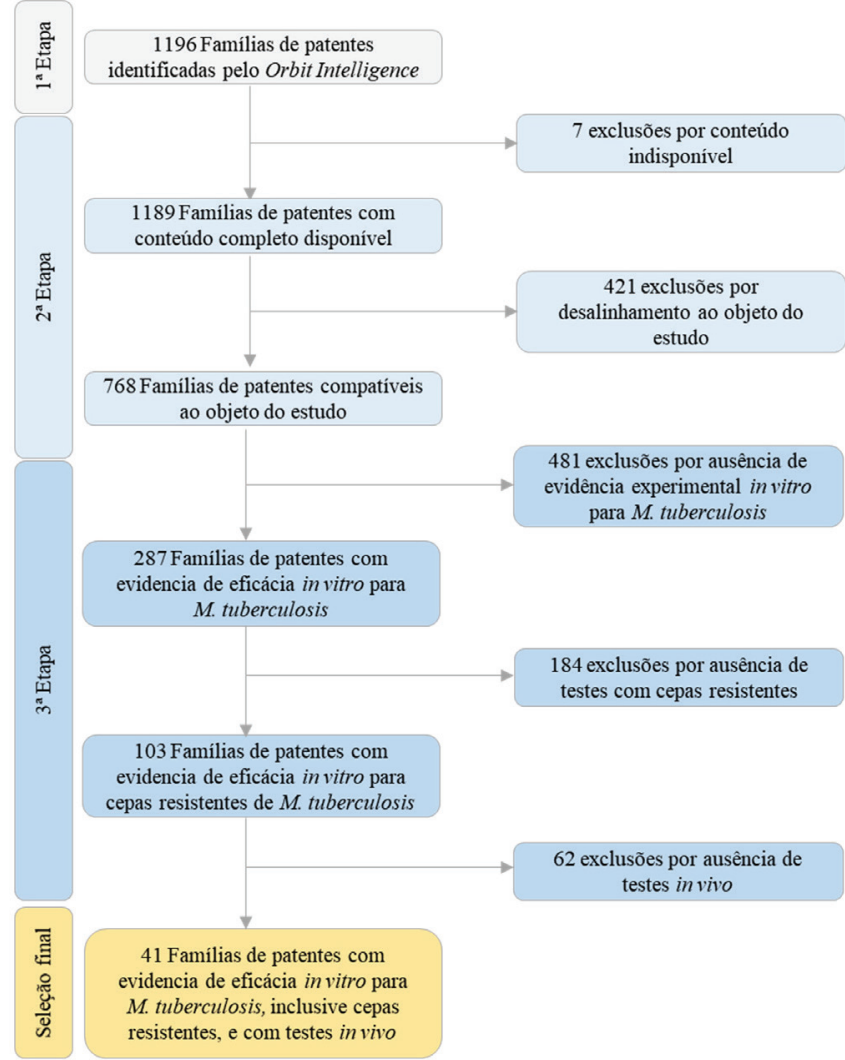

Figura 1. Fluxo metodológico para identificação de novas tecnologias desenvolvidas para o tratamento da TB

experimental in vitro para $M$. tuberculosis, 184 famílias por ausência de testes com cepas resistentes do microrganismo e 62 famílias por ausência de testes in vivo.

Após todas as etapas do fluxo metodológico, foram identificadas 41 famílias de patente de novas tecnologias desenvolvidas para o tratamento da TB com atividade experimental in vitro para cepas resistentes de $M$. tuberculosis e testes in vivo, depositadas de janeiro de 2015 a fevereiro de 2017. É necessário considerar que os documentos de patente somente são publicados 18 meses após o depósito, assim a busca realizada em agosto de 2019 somente detectou documentos depositados até fevereiro de 2017.

Com relação ao status legal da proteção patentária para as 41 novas tecnologias identificadas neste estudo, para 37 (90\%) delas há algum tipo de proteção ou expectativa em pelo menos um território ou país. Nesse elenco, 23 (56\%) aguardam análise, enquanto 14 (34\%) já tiveram proteção patentária concedida, indicando que os critérios de patenteabilidade foram alcançados.

Para as 4 (10\%) famílias restantes, 3 delas expiraram e 1 foi indeferida, ou seja, a proteção foi encerrada por um ou mais motivos, como por exemplo, falta de novidade. Nesse sentido, o conhecimento divulgado nesses documentos de patentes pode ser explorado livremente, sem qualquer necessidade de contrapartida, em qualquer lugar do mundo.

As novas tecnologias desenvolvidas estão protegidas principalmente na China (19), no Escritório de Patente Europeu (sigla em inglês, $E P O)$ (15) - que abrange todos os estados membros da União Europeia, além de Albânia, Suíça, Reino Unido, Islândia, Mônaco, Macedônia do Norte, Noruega, Sérvia, Liechtenstein e São Marinho - nos Estados Unidos da América (EUA) (8), seguidos por Brasil (6), Rússia (6) e Índia (4). A Figura 2 ilustra geograficamente o número de patentes vivas, identificadas neste estudo, protegidas nos vários escritórios nacionais. 


\section{Depósito de patentes por país de proteção}

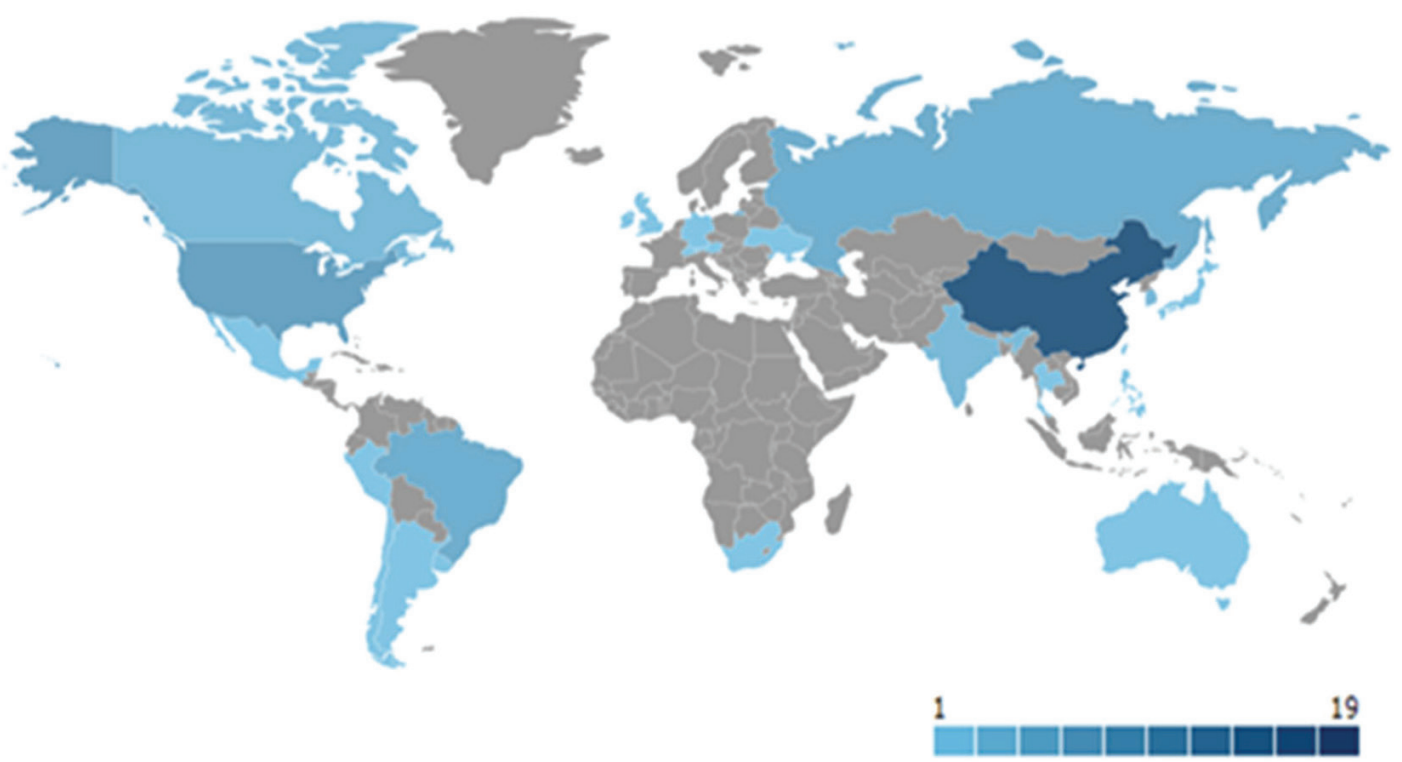

Figura 2. Principais países de depósito das patentes de novas tecnologias desenvolvidas para o tratamento da TB

A Figura 2 indica a estratégia de proteção dos detentores das tecnologias, ao identificar os mercados-alvo de proteção. De forma geral, é comum que responsáveis pelo desenvolvimento de novas tecnologias depositem suas patentes em países considerados estratégicos para suas invenções, países com grandes mercados consumidores, economicamente importantes, bem como em países com licenciadores em potencial, como em áreas geográficas de produção fabril de seus concorrentes. ${ }^{12}$

No caso da TB, a doença afeta o mundo todo, porém 30 países concentram $87 \%$ dos casos no mundo. A maioria dos casos registrados em 2018 ocorreram no Sudeste Asiático (44\%), África (24\%) e Pacífico Ocidental (18\%), com porcentagens menores no Mediterrâneo Oriental (8\%), nas Américas (3\%) e Europa (3\%). Ocorreram cerca de meio milhão de novos casos de $\mathrm{TB}$ resistentes à rifampicina, dos quais $78 \%$ apresentavam TB multirresistente (quando há resistência à rifampicina e à isoniazida). Os novos casos de TB resistente se concentraram na África do Sul (3\%), Índia (27\%), China (14\%) e Rússia (9\%). ${ }^{1}$ Contudo, a despeito da alta carga da doença, alguns países podem não ter despertado o interesse de proteção para as novas tecnologias por parte de seus detentores, devido à ausência de poder de compra, desinteresse político, ou por fragilidade jurídica no âmbito da propriedade intelectual.

O Brasil é o quinto país no ranking entre os territórios com mais tecnologias depositadas. Em um país com um alto ônus da doença e em que esforços foram feitos para combater a TB, essa posição pode ser considerada baixa. No entanto, tal cenário pode ser visto sob um viés vantajoso posto que, como parte dessas novas tecnologias não está protegida no país, não haveria infração de patente na exploração de tal conhecimento, abrindo caminho para a possibilidade de desenvolvermos tais tecnologias por imitação. Muitos processos de desenvolvimento econômico e recuperação tecnológica começaram por meio da produção de cópias de produtos inovadores. ${ }^{13} \mathrm{Ou}$ seja, a cópia desenvolvida é considerada nova apenas nos países que adotam o novo produto, mas não é necessariamente nova no estado da arte. A imitação pode reduzir os custos e os riscos da descoberta e permitir a criação de recursos inovadores. Além disso, a utilização do conhecimento divulgado nas patentes pode reduzir o tempo em pesquisa, uma vez que o desenvolvimento de novas tecnologias pode ser iniciado a partir das invenções que estão em domínio público. ${ }^{14}$ E todas as possibilidades de redução das incertezas e a otimização dos resultados visando atender às necessidades de saúde da sua população e estimular o processo de inovação na área da saúde devem ser consideradas. ${ }^{15}$

A Tabela 1 mostra todos os depositantes responsáveis pelas novas tecnologias identificadas neste estudo pelos 8 países / escritórios com maior incidência de depósito. A intensidade da cor reflete o número de famílias no cruzamento.

As instituições responsáveis pelo patenteamento de novas tecnologias de TB são principalmente as universidades (14). Há também empresas privadas (8), instituições de pesquisa (6) e fundações de apoio à pesquisa (3). A maioria está localizada na China (12) e EUA (7), mas existem instituições na Rússia (4), Europa (3) e Brasil (2), além de Canadá (1), Austrália (1) e República da Coréia (1).

Embora existam questionamentos quanto ao uso da patente como indicador de inovação, ${ }^{16}$ tradicionalmente os países inovadores reconhecidos como potências mundiais possuem um número expressivo de pedidos de patentes. ${ }^{17,18}$ Assim, um número muito pequeno de pedidos de patentes de instituições brasileiras (2) sugere que, para uma doença com alta carga no país, o Brasil deveria dirigir maiores esforços na promoção de P\&D voltada para o desenvolvimento de produtos ou processos inovadores.

Dentre os depositantes brasileiros estão a entidade privada União Brasileira de Educação e Assistência mantenedora da Pontifícia Universidade Católica do Rio Grande do Sul (PUCRS) e a Universidade Estadual Paulista Júlio de Mesquita Filho (UNESP), universidade pública de São Paulo.

No Brasil, de modo geral, universidades e instituições de pesquisa têm liderado os depósitos de patentes de residentes no país, estimuladas pelas fundações estaduais de apoio à ciência e tecnologia. No entanto, o destaque das universidades revela a baixa atividade de P\&D das empresas brasileiras. ${ }^{16}$

No segmento farmacêutico este cenário já é conhecido. Embora seja um setor intensivo em $P \& D$ e que valoriza o controle de ativos intangíveis vinculados à inovação, especialmente as patentes, o setor farmacêutico nacional apresenta baixo poder de inovação quando comparado aos países europeus, aos EUA e a outros países 
Tabela 1. Instituições detentoras das patentes de novas tecnologias para TB por país/ escritório a de proteção

\begin{tabular}{|c|c|c|c|c|c|c|c|c|}
\hline Instituição detentora (país) / País de proteção & $\mathrm{CN}$ & WO & US & EP & BR & RU & IN & $\mathrm{KR}$ \\
\hline Institute of Medicinal Biotechnology Chinese Academy of Medical Sciences (CN) & 10 & & & & & & & \\
\hline Zhejiang Starry Pharmaceutical (CN) & 7 & & & & & & & \\
\hline Institute of Materia Medica Chinese Academy of Medical Sciences (CN) & 2 & 2 & & & & & & \\
\hline Yuria Pharm (UA) & & 3 & & & & & & \\
\hline Beijing Tuberculosis and Thoracic Tumor Research Institute $(\mathrm{CN})$ & 2 & & & & & & & \\
\hline Glaxosmithkline Intellectual Property Development Limited (GB) & 1 & 2 & 1 & 1 & 1 & & 1 & 1 \\
\hline Johns Hopkins University (US) & 1 & 2 & 1 & & & & & \\
\hline Cisen Pharmaceutical $(\mathrm{CN})$ & & 1 & 1 & 1 & 1 & 1 & 1 & \\
\hline Curtin University (AU) & 1 & 1 & & & & & & \\
\hline East China Normal University $(\mathrm{CN})$ & 1 & 1 & & & & & & \\
\hline L'Ecole polytechnique fédérale de Lausanne $(\mathrm{CH})$ & & 1 & 1 & 1 & & & & \\
\hline
\end{tabular}

Federal State Budgetary Institution of Science Institute (RU)

Federal'noe Gosudarstvennoe Byudzhetnoe Obrazovatel'noe Uchrezhdenie (RU)

Guangzhou Institute of Respiratory Medicine $(\mathrm{CN})$

Guizhou University $(\mathrm{CN})$

Joint Stock / Pharmasyntez (RU)

Konkuk University Industrial Cooperation Corp (KR)

Leland Stanford Junior University (US)

The People's Liberation Army (PLA) No. 309 Hospital (CN)

Qifang Pharmaceutical Industry (CN)

Research Foundation of Suny (US)

Rutgers University (US)

Shangai Jia Tan Pharmatec $(\mathrm{CN})$

Shanghai Sun Sail Pharmaceutical Science \& Technology (CN)

South Ural State University (RU)

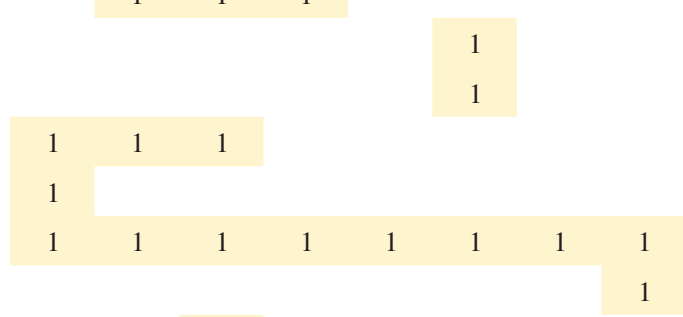

Texas A\&M University (US)

União Brasileira de Educação e Assistência Mantenedora da PUCRS (BR)

Universidade Estadual Paulista Júlio de Mesquita Filho (BR)

University of British Columbia (CA)

University of Illinois at Chicago (US)

University System of Georgia Valdosta State University (US)

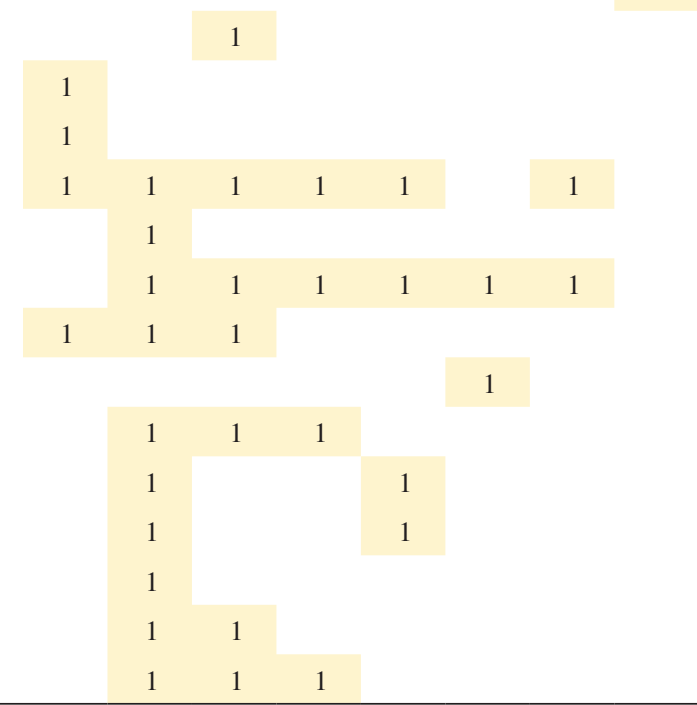

${ }^{\mathrm{a}} \mathrm{AU}=$ Austrália; $\mathrm{BR}=$ Brasil; $\mathrm{CA}$ = Canadá; $\mathrm{CH}$ = Suíça; $\mathrm{CN}$ = China; $\mathrm{EP}$ = EPO; GB = Reino Unido; IN = Índia; $\mathrm{KR}$ = República da Coréia; RU = Rússia; UA = Ucrânia; US = EUA; WO = Organização Mundial de Propriedade Intelectual.

emergentes, como China e Índia, como consequência da estratégia das empresas brasileiras se especializarem na produção de genéricos. ${ }^{19}$ Atividades concentradas em P\&D de novos medicamentos, sejam por síntese química ou rota biotecnológica, demandam competências raramente observadas nas empresas e instituições científico-tecnológicas nacionais. ${ }^{20}$

É importante considerarmos também que no Brasil não há uma "cultura" de proteção à propriedade intelectual, realidade essa que se reflete em deficiências nos pedidos de patentes, com prejuízo para os depositantes e para o sistema. Entraves como ausência de financiamento para transformação das invenções em inovações e o chamado backlog também comprometem o patenteamento no país. O prazo médio para decisão da concessão das patentes no Brasil é de 10,2 anos, enquanto no Japão é de 1,3 anos e nos EUA e União Europeia é de 2,2 anos. Há consenso de que essa demora traz enormes prejuízos para todas as partes interessadas, para a economia e principalmente para a sociedade brasileira. ${ }^{16}$ Todos os documentos de patentes identificados neste estudo que estão depositados no Brasil (6), estão aguardando análise pelo órgão responsável.

Algumas ações, buscando colocar o Brasil no cenário internacional de inovação, têm sido tomadas. O mais recente passo foi a aprovação da Lei no 13.243/2016 conhecida como "novo marco legal da inovação" que estabeleceu medidas de incentivo à inovação e à pesquisa científica e tecnológica do país. ${ }^{21}$

A Tabela 1 ainda permite verificar que os principais depositantes de novas tecnologias estão localizados nos principais países de depósito dessas tecnologias (Figura 2), refletindo a tendência dos desenvolvedores de novas tecnologias a protegerem frequentemente suas patentes em seu país de origem. Nota-se, contudo, que algumas instituições buscaram diversificar os países onde buscam proteção, realizando depósito em ao menos um outro país.

Outra estratégia de patenteamento observada foi o depósito de pedido de patente internacional via Tratado de Cooperação em Matéria de Patentes (sigla em inglês, $P C T$ ) na Organização Mundial 
da Propriedade Intelectual (sigla em inglês, WIPO). O PCT é um pacto internacional com mais de 150 estados contratantes que permite a proteção de patentes para uma invenção simultaneamente em um grande número de países, registrando um único pedido de patente internacional postergando a apresentação de vários pedidos de patentes nacionais ou regionais. ${ }^{22} \mathrm{O}$ índice baseado no depósito de patentes na WIPO indica que os responsáveis têm interesse na internacionalização das tecnologias desenvolvidas e, como os custos de depósito no exterior podem ser substanciais, patentes para as quais os solicitantes buscam proteção internacional são consideradas mais valiosas.

Assim, relaciona-se o grau de internacionalização da proteção patentária de uma tecnologia à sua relevância. A prática comum de mercado é que, ao perceberem que detém uma grande inovação, os detentores de uma nova tecnologia solicitam proteção em muitos países/territórios, mesmo cientes dos altos custos para tradução, processamento e representação em cada país/território peticionado. A proteção patentária internacional pode incorrer em elevados gastos ao longo de toda a vida da patente. Gastos iniciais com taxas para o depósito de um pedido de patente internacional, via PCT, podem variar entre 7 e 14 mil reais, e ainda devem ser acrescidos dos honorários de representação. Ademais, custos de tradução para outras línguas podem variar de centenas a milhares de reais dependendo da disponibilidade de profissionais tradutores.

Trata-se, portanto, de uma decisão estratégica que pondera a relação custo de proteção versus benefício de proteção. Por outras vezes, dado os custos envolvidos na proteção em diversos países/territórios, como acima discutido, para muitas instituições a internacionalização pode tornar-se inviável economicamente mesmo para invenções potencialmente promissoras. O que significa que a tecnologia é protegida somente no país sede da empresa, provavelmente onde foi gerado o $\mathrm{P} \& \mathrm{D}$, deixando a tecnologia em domínio público em todos os demais países. A proteção no Brasil, nesse aspecto, é extremamente atrativa para os detentores da tecnologia, posto que, para um inventor individual ou uma Instituição de Ciência e Tecnologia, a taxa para o depósito de um pedido de patente é reduzida em $60 \%$, restando em $\mathrm{R} \$ 70,00$. Pode-se inferir, portanto, que durante toda a vida de uma patente, há ainda outras taxas para o processamento. Contudo, não devem ser consideradas como impeditivas ao patenteamento pois não implicam em custos excessivos.

Assim, as informações da Tabela 1 nos permitem identificar tanto as novas tecnologias com alto grau de internacionalização, portanto relevantes, quanto as novas tecnologias sem proteção no Brasil, estando, deste modo, livres para serem desenvolvidas e exploradas no país.

Além do exposto, a identificação das instituições detentoras das patentes das novas tecnologias pode também direcionar estrategicamente os melhores parceiros para construção de redes de cooperação em $\mathrm{P} \& \mathrm{D}$ para combate à $\mathrm{TB}$. As redes colaborativas são consideradas promotoras de inovação para organizações de CT\&I. ${ }^{23}$

\section{Novas tecnologias identificadas}

Apesar do notável crescimento dos produtos da biodiversidade entre os novos medicamentos aprovados, ${ }^{24} 37$ (90\%) das 41 novas tecnologias identificadas neste estudo são produtos obtidos por síntese química. Outras $2(5 \%)$ tecnologias são preparações de medicina tradicional chinesa e $2(5 \%)$ são sistemas de liberação de fármacos.

Entre as 37 novas tecnologias obtidas por síntese química, a maioria das patentes identificadas apresentou a estrutura química das substâncias ou séries de substâncias ativas. As estruturas e os grupos químicos são ilustrados na Tabela 2 .

A maioria das novas tecnologias obtidas por síntese química
(26; 70\%) consiste em substâncias heterocíclicas, demonstrando a importância dos heterociclos na química medicinal. ${ }^{25}$ Observa-se que há $23(62 \%)$ heterociclos com pelo menos um átomo de nitrogênio inserido no ciclo, enquanto apenas $6(20 \%)$ dos heterociclos apresentados nas patentes possuem / podem possuir átomos de enxofre no anel e 8 (30\%) possuem / podem possuir átomos de oxigênio, salientando a relevância da atividade biológica dos heterociclos nitrogenados, que são os componentes estruturais mais presentes nos produtos farmacêuticos. ${ }^{26}$

Outro fato a ser destacado é a presença de pelo menos um átomo de flúor ou grupo trifluorometil em 8 (21\%) das estruturas. A presença de átomos de flúor ligados a anéis aromáticos, entre outras consequências, pode levar ao aumento da lipofilicidade de uma molécula. ${ }^{27}$ Dessa forma, é possível que a substituição por átomos de flúor seja um fator interessante no desenvolvimento de medicamentos contra micobactérias.

Além disso, analisando as estruturas da Tabela 2, é possível ver que uma das novas tecnologias desenvolvidas envolve um estereoisômero de cloroquina. Sabe-se que a molécula não é nova. A cloroquina é um agente antimalárico antigo. ${ }^{28} \mathrm{~A}$ novidade é que a forma enantiomericamente pura $(\mathrm{R})$-cloroquina teria maior eficácia contra M. tuberculosis do que sua mistura racêmica.

As estruturas identificadas são de diferentes grupos químicos. No entanto, é possível destacar alguns deles: tiouréia, hidrazina, quinolina, quinolona, imidazole e indol, dentre outros. Atualmente, alguns dos medicamentos preconizados para tratamento da TB multirresistente são quinolonas ${ }^{2} \mathrm{e}$ a atividade antituberculose já evidenciada para diversos compostos dos grupos identificados ${ }^{29-33}$ confere relevância às novas tecnologias identificadas, evidenciando-as como potenciais candidatas a novos medicamentos contra a TB.

Além dos produtos obtidos por síntese química, foram identificadas 2 novas tecnologias envolvendo preparações de medicina tradicional chinesa: CN104689129 (Anti-tuberculous traditional Chinese medicine fritillary and burdock fruit tuberculosis-eliminating extractive and application) e CN104771494 (Compound traditional Chinese medicine preparation for treating tuberculosis). O tratamento da TB através de infusões, macerações, tinturas e decocções de partes de plantas medicinais é realizado há séculos em diferentes sistemas tradicionais de medicina no mundo, incluindo o chinês, o africano e o indiano ou Ayurveda. ${ }^{34}$

De fato, muitos extratos de diversas espécies de vegetais utilizados para TB exibem atividades antimicobacterianas significativas in vitro. ${ }^{35}$ Contudo, embora utilizados com considerável eficácia, as doses terapêuticas e seguras ainda devem ser estabelecidas para a maioria das formulações. ${ }^{34}$ Assim, são necessários esforços voltados para identificação das substâncias ativas, do mecanismo de ação e do potencial terapêutico das novas tecnologias desenvolvidas.

Foram identificadas ainda 2 novas tecnologias envolvendo sistemas de liberação de fármacos: EP3331504 (Tablet composition for anti-tuberculosis antibiotics) e WO201715736 (Method for obtaining lipid nanostructures, thus obtained lipid nanostructures and use thereof). De forma geral, esses sistemas objetivam ampliar o tempo de liberação do fármaco no organismo permitindo redução da toxicidade e das reações adversas, bem como a diminuição do número de doses administradas, conferindo maior comodidade posológica ao tratamento. Sistemas fundamentados em nanotecnologia têm se mostrado capazes de direcionar o fármaco para que ele seja disponibilizado diretamente nos órgãos, tecidos e células afetados, conferindo maior seletividade e menor toxicidade durante a terapia medicamentosa. ${ }^{36}$ Novos sistemas de liberação de fármacos aplicados ao tratamento farmacológico da TB podem contribuir para aumento da adesão ao tratamento, aumentando as possibilidades de cura e reduzindo o risco de resistência aos medicamentos. 
Tabela 2. Estruturas e grupos químicos das 37 novas tecnologias obtidas por síntese química identificadas nos documentos de patente

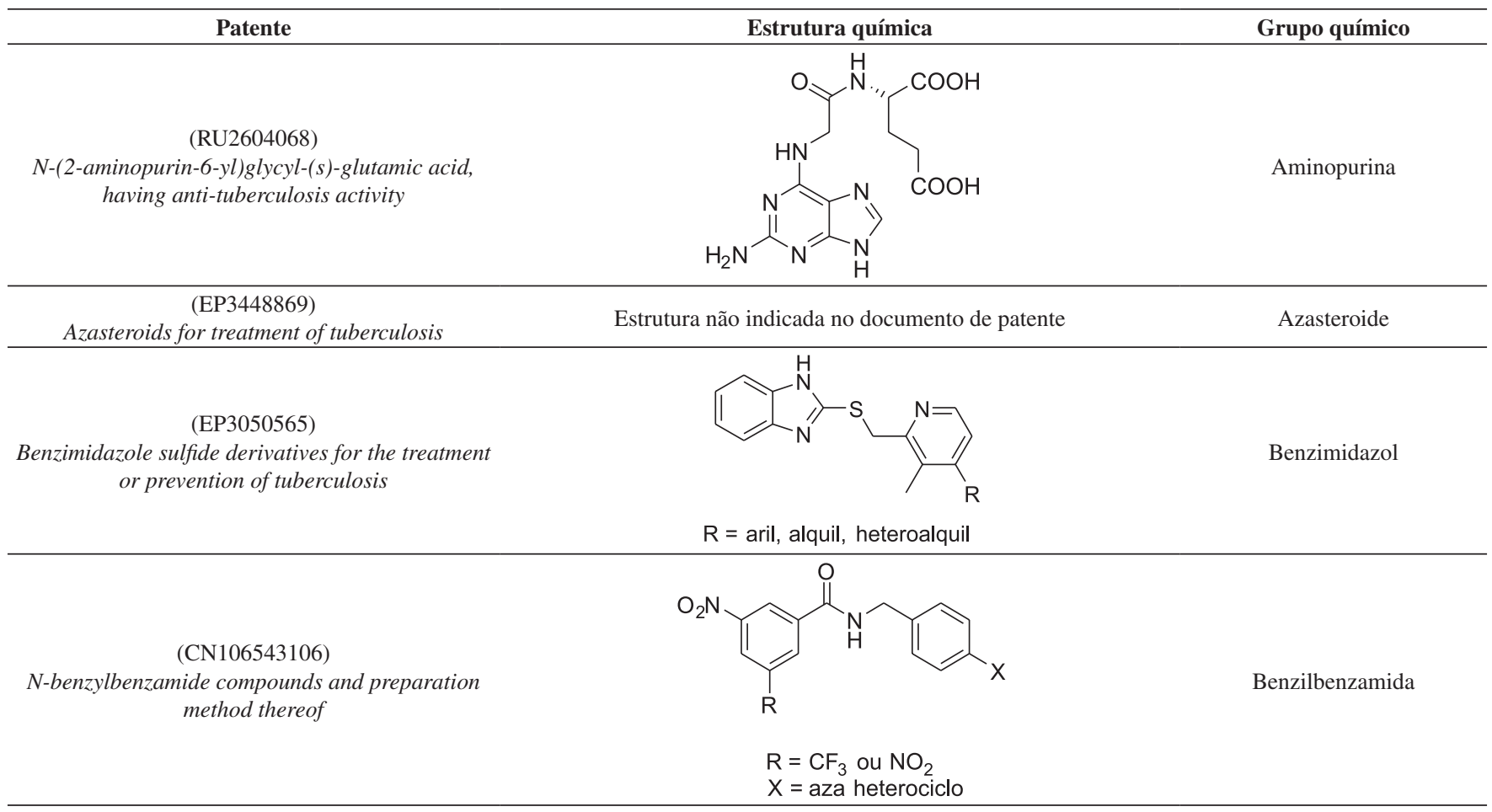

(EP3285873)

Substituted benzofuran derivatives as novel antimycobacterial agents

(CN105669664)

Benzothiazine-4-ketone compounds containing basic nitrogen heterocyclic fragments and preparing methods of benzothiazine-4-ketone compounds

\section{(CN105622596)}

Benzothiazine-4-ketone compound containing alkoxyimino azacyclo-fragments and preparation method of benzothiazine-4-ketone compound

(CN108440446)

Benzothiazine-4-acetone compound containing oximido fragment and preparation method thereof

Benzothiazine-4-one compound containing alkaline bridge ring fragment and preparation method thereof

Estrutura não indicada no documento de patente

Benzofurano

Estrutura não indicada no documento de patente

Benzotiazina<smiles>[R]c1nc(=O)c2c([R])c([R])c([R])c([R])c2s1</smiles>

Benzotiazina

$\mathrm{R}=\mathrm{CF}_{3}, \mathrm{NO}_{2}$, alquil, aril<smiles>[R]ON=C1CCC(CN2CCN(c3nc(=O)c4cc([X])cc([N+](=O)[O-])c4s3)CC2)CC1</smiles>

Benzotiazina

$$
\mathrm{X}=\mathrm{NO}_{2}, \mathrm{CF}_{3}
$$

$\mathrm{R}=$ alquil, aril<smiles>[R]c1nc(=O)c2cc(C(F)(F)F)cc([N+](=O)[O-])c2s1</smiles>

Benzotiazina-4-ona

$\mathrm{R}=$ alquil, aril, heteroaril

(RU2663848)

5-fluoro-2-(4-ethoxycarbonyldipiperazine-1-yl)-1,3benzothiazine-4-one, which has anti-tuberculosis activity

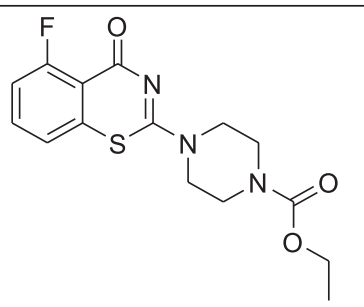


Tabela 2. Estruturas e grupos químicos das 37 novas tecnologias obtidas por síntese química identificadas nos documentos de patente (cont.)

Patente
(RU2683573)
$\begin{gathered}\text { Anti-tuberculosis agent based on } \\ \text { n-[4-(4-aminobenzsulfanyl)-phenyl-2- } \\ \text { benzoylaminobenzamide with low toxicity }\end{gathered}$
(US20180111913)
Substituted benzofuran derivatives as novel
antimycobacterial agents

(WO2019120210)

Benzofuran, coumestans derivatives, preparation method and use thereof<smiles>[R]C(=O)c1c([R])[X]c2c([R])c([R])c([R])c([R])c12</smiles>

Benzofurano

$\mathrm{R}=$ alquil, aril,

carbonilados, heterociclos

$\mathrm{X}=\mathrm{O}$ ou $\mathrm{N}$ ou $\mathrm{S}$

(KR101923514)

Composition comprising Phloretin or as active ingredients for preventing or treating of Tuberculosis

(CN108059627)

Anti-mycobacterium tuberculosis compound as well as preparation method and application thereof

Inhibitors of drug-resistant mycobacterium tuberculosis

WO201946465)

Therapeutic indoles

Sanfetrinem or a salt or ester thereof for use in treating mycobacterial infection

\section{$\mathrm{HO}$}<smiles>Cc1ccc(/C=N/NC(N)=S)o1</smiles>

Flavonoide

Furano e tioamida<smiles>[R]CCN([R6])C(=O)C1[Y]=c2c([R4])c([R])c([R])c([R])c2=[X]1</smiles>

Indol
Indo

$\mathrm{R}=\mathrm{H}$, halogênio

$\mathrm{COOH}, \mathrm{COOEt}$

COOAr, CONR, alquil, sulfonamida

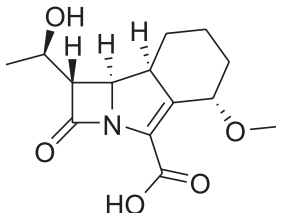


Tabela 2. Estruturas e grupos químicos das 37 novas tecnologias obtidas por síntese química identificadas nos documentos de patente (cont.)

\begin{tabular}{ccc}
\hline Patente & Grupo químico \\
(CN107674014) \\
$\begin{array}{c}\text { 3,5-dinitrobenzene formamide compound containing } \\
\text { isoindoline fragment and preparation method } \\
\text { thereof }\end{array}$
\end{tabular}

(CN106588916)

$N$-(phenoxyethyl)imidazo [1,2-a] pyridine-3-amide compound and preparation method thereof

(CN108358917)

Imidazo[1,2-a]pyridine-3-amide compound containing alkaline fused ring fragment and preparation method thereof<smiles>[Y]c1ccc(OCCNC(=O)c2c([R2])nc3ccc([R])cn23)cc1</smiles>

Imidazol

$$
\begin{aligned}
& \mathrm{R}=\mathrm{H}, \mathrm{CH}_{3}, \mathrm{CH}_{2} \mathrm{CH}_{3} \\
& \mathrm{Y}=\text { aril } \\
& \mathrm{n}=0 \text { ou } 1
\end{aligned}
$$

(CN109503631)

Diazospiro fragment-containing imidazo[1,2-alpha] pyridine-3-amide compound and preparation method and application thereof

(WO201691207)

Nitroimidazole compound, preparation method therefor and use thereof in drug manufacturing

(WO201662151)

Pyrazolo[1,5-a]pyridine compounds and use thereof

(EP3170810)

Pyridine derivatives and anti-mycobacterial use thereof
Estrutura não indicada no documento de patente<smiles>[R][IH][Y]([H])([H])c1[X]c([R])c(C(=O)N([R])[C@H]2COc3nc([N+](=O)[O-])cn3C2)cn1</smiles>

$$
\begin{aligned}
& n=1 \text { a } 4 \\
& X=C \text { ou } N \\
& L=O, S \text { ou } N H \\
& r=\text { alquil ou aril }
\end{aligned}
$$

Imidazo [1,2-a] piridina -3amida
Nitroimidazol

Pirazol

$$
\mathrm{R}=\mathrm{H} \text {, alquil, aril }
$$

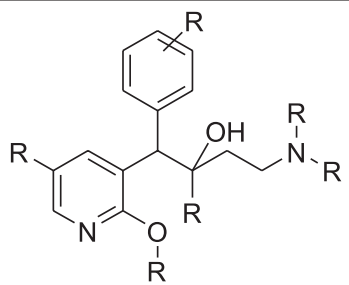

Piridina 
Tabela 2. Estruturas e grupos químicos das 37 novas tecnologias obtidas por síntese química identificadas nos documentos de patente (cont.)

Patente
(WO2018116260)
$\begin{gathered}\text { effect } \\ \text { Pharmaceutical composition with anti-tubercular }\end{gathered}$
(EP3432887)
Antituberculosis agent
$\begin{gathered}\text { Pyrrole-2-formamide compound, and preparation } \\ \text { method therefor and applications thereof }\end{gathered}$
(WO201942267)

(US20160113919)

Chloroquine stereoisomer for treating tuberculosis related diseases<smiles>CCN(CC)CCCC[C@H](C)Nc1ccnc2cc(Cl)ccc12</smiles>

Quinolina
(WO2016201541)

Compound, method for producing the compound, pharmaceutical composition, use of the compound

for preparing a medicinal drug for treating a disorder caused by bacteria of the genus mycobacterium and method for treating a disorder caused by bacteria of the genus mycobacterium

(CN104892600)

7-(3-aminomethyl-4-substituted-benzyloxyimino1-pyrrolidinyl)naphthyridinone carboxylic acid compounds

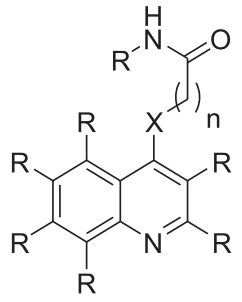

$\mathrm{R}=\mathrm{H}$, alquil, aril

$\mathrm{X}=\mathrm{CH}_{2}, \mathrm{O}, \mathrm{N}$ ou $\mathrm{S}$

$\mathrm{n}=0-3$<smiles>[R]Cc1ccccc1CO/N=C1/CN(C2N=c3c(c(=O)c(C(=O)O)cn3[C@H](C)CF)=CC2[R])C[C@@H]1CN</smiles>

$\mathrm{R}$ = halogênio, metil, metóxi, dimetóxi, dioximetileno

(CN108727332)

Propylene tethered ciprofloxacin-isatin hybrids as well as synthetic method and application thereof

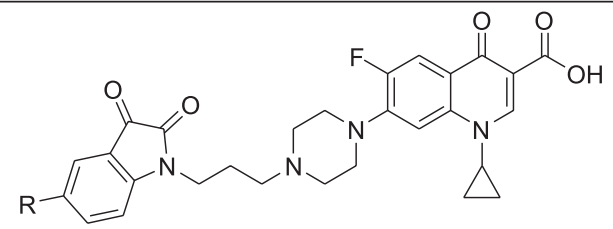


Tabela 2. Estruturas e grupos químicos das 37 novas tecnologias obtidas por síntese química identificadas nos documentos de patente (cont.)

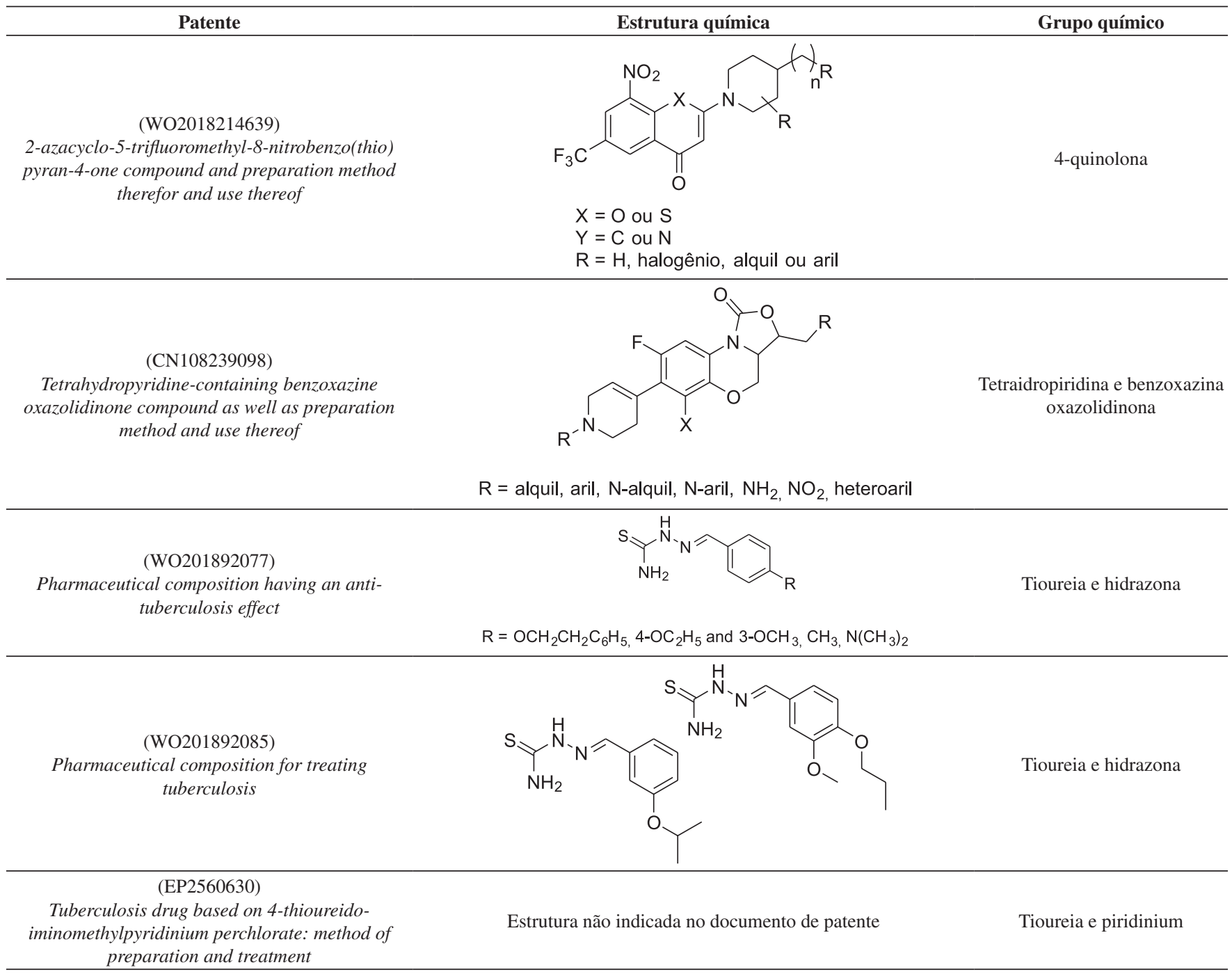

\section{Alvos biológicos}

Entre os documentos de patente analisados neste estudo, $6(15 \%)$ dos documentos apresentaram evidências do alvo biológico das novas tecnologias, todas relacionadas à inibição da biossíntese da parede celular de M. tuberculosis.

As micobactérias em geral, incluindo $M$. tuberculosis, são cercadas por um envelope celular de membrana dupla que as torna intrinsecamente resistentes a muitos antibióticos. Especificamente, a membrana externa é composta por ácidos graxos ramificados de cadeia longa chamados ácidos micólicos, que são agrupados para produzir uma bicamada com baixa permeabilidade. ${ }^{37}$ Sabe-se que os ácidos micólicos são críticos para a viabilidade e virulência de M. tuberculosis, tornando sua via de síntese um alvo viável para o desenvolvimento de novos medicamentos para TB. A biossíntese de ácido micólico envolve a ação de mais de 20 enzimas que fazem parte de diferentes complexos multi-enzimas. ${ }^{38} \mathrm{~A}$ maioria dos medicamentos utilizados clinicamente para tratar a TB tem como alvo a síntese macromolecular. ${ }^{39}$ Fármacos eficazes no tratamento da TB, como isoniazida, etambutol, etionamida, carbapenêmicas e delamanida, são direcionados para a parede celular. ${ }^{8}$

Três das novas tecnologias analisadas demonstraram a inibição de uma enzima essencial na síntese de ácido micólico conhecida como policetídeo sintase Pks13, necessária para a formação da parede celular do M. tuberculosis (EP3285873; US20180111913; e WO2019120210). Essa enzima é considerada um alvo promissor. ${ }^{40}$

Uma tecnologia direcionou como seu alvo de ação a proteína da membrana micobacteriana conhecida como MmpL3 (WO2015164482). A MmpL3 é necessária para a translocação de ácidos micólicos na forma de monomicolatos no citoplasma para o espaço periplásmico, onde os ácidos micólicos podem ser usados para montar a membrana externa das micobactérias. ${ }^{41}$ Possivelmente a MmpL3 desempenha um papel no transporte de outros componentes lipídicos importantes para a parede celular. Assim, a capacidade de bloquear a ligação e o transporte desses lipídios via MmpL3 tornou essa proteína de membrana um alvo emergente para a terapia da TB. ${ }^{42}$

Outra tecnologia apresentou como possível alvo a proteína transportadora de $M$. tuberculosis $\beta$-cetoacil acil sintase III (mtKASIII) (KR101923514). MtKASIII é uma enzima de condensação responsável pelo início da biossíntese de ácidos graxos. Uma nova tecnologia que se liga com alta afinidade, causando sua inibição, contribui para o aumento da permeabilidade da membrana micobacteriana, tornando-a um alvo potencial para a ação de novos agentes antituberculose. Também se demonstrou que a mesma tecnologia inibe a sinalização da proteína quinase ativada por mitogênio p38 (MAPK) e a sinalização quinase regulada por sinal extracelular $(E R K)$. A modulação da expressão de citocinas pró-inflamatórias via inibição da sinalização $p 38 M A P K / E R K$ suprime a liberação de citocinas 
pró-inflamatórias, como interleucinas (IL) $I L-1 \beta, I L-6$ e $I L-12$ e fator de necrose $(T N F)-\alpha .{ }^{43}$ Estudos sugerem que a inibição dessas vias pró-inflamatórias pode limitar o dano tecidual característico da TB. ${ }^{44}$

Finalmente, uma tecnologia direcionou a inibição da $\beta$-cetoacilACP sintase (KasA), um componente essencial da biossíntese de ácidos graxos de M. tuberculosis ${ }^{8}$ reforçando os esforços centrados no desenvolvimento de novos inibidores da via de biossíntese de ácido micólico (WO201946465).

Assim, ainda que apenas $6(15 \%)$ das novas tecnologias tenham apresentado evidências do alvo biológico, é necessário ponderarmos que documentos de patentes tendem a disponibilizar o mínimo de dados experimentais possível. Dessa forma, a existência de resultados de atividade experimental in vitro para cepas resistentes de $M$. tuberculosis e testes in vivo, divulgando adicionalmente a evidencia do mecanismo de ação, torna a informação dessas patentes muito atrativa.

\section{CONCLUSÃO}

Por meio de uma prospecção tecnológica, utilizando patentes como fonte de informação, foram identificadas 41 novas tecnologias desenvolvidas para o tratamento da TB, com evidências experimentais de eficácia in vitro especificamente para a M. tuberculosis, incluindo cepas resistentes, e ensaios in vivo.

Para a maior parte das patentes identificadas, há algum tipo de proteção ou expectativa em pelo menos um território ou país onde estas foram depositadas. A proteção a essas patentes foi requerida principalmente na China, União Europeia, EUA, Brasil, Rússia e Índia, todos países com mercados promissores, com licenciadores em potencial e sólida regulamentação em propriedade intelectual.

As instituições responsáveis pelo patenteamento das novas tecnologias de TB são universidades, empresas privadas e instituições de pesquisa localizadas na China, EUA, Rússia, Europa e Brasil. A maioria das patentes foi internacionalizada, reforçando a relevância das novas tecnologias identificadas.

As novas tecnologias envolvem principalmente produtos de síntese química, principalmente heterociclos, em maior parte nitrogenados. Embora a biotecnologia e a nanotecnologia sejam consideradas nichos estratégicos para a indústria farmacêutica, os resultados deste estudo reforçam que a direção das atividades de $\mathrm{P} \& \mathrm{D}$ e produção de medicamentos do país deve não apenas levar em consideração as tendências tecnológicas do mercado, mas deve estar alinhada principalmente com as necessidades da população.

Embora as descrições e reivindicações de patentes sejam formatadas de maneira bastante diferente de um artigo científico, entendemos que a valorização das patentes como fonte de informação pode acelerar o processo de descoberta de novos medicamentos para o combate à TB e TB resistente. Em vista disso, o uso de patentes como fonte de informação, em combinação com o uso de softwares e técnicas de mineração de dados, pode otimizar o desenvolvimento de novos medicamentos para o tratamento da TB, promovendo inovação, gerando riquezas para o país e respostas para as necessidades em saúde da população brasileira.

\section{REFERÊNCIAS}

1. World Health Organization. Global tuberculosis report 2019. Geneva, 2019, disponível em https://www.who.int/tb/publications/global_report/ en/, acessado em junho 2020.

2. Brasil. Ministério da Saúde. Secretaria de Vigilância em Saúde. Departamento de Vigilância das Doenças Transmissíveis. Manual de Recomendações para o Controle da Tuberculose no Brasil. Brasília, 2019, disponível em https://sbpt.org.br/portal/wp-content/ uploads/2019/06/manual_recomendacoes_tb_2ed_atualizada_8maio19. pdf, acessado em junho 2020.

3. Bloom, B.R.; Atun, R.; Cohen, T.; Dye, C.; Fraser, H.; Gomez, G. B.; Knight G.; Murray M.; Nardell, E.; Rubin, E.; Salomon, J.; Vassall, A.; Volchenkov, G.; White, R.; Wilson, D.; Yadav, P. Em Major Infectious Diseases; Holmes, K.K.; Bertozzi, S.; Bloom, B. R.; Jha, P., eds.; Washington (DC): The International Bank for Reconstruction and Development / The World Bank, 2017, cap. 11.

4. Organização das Nações Unidas. Transformando Nosso Mundo: A Agenda 2030 para o Desenvolvimento Sustentável. Última edição, 2015, disponível em https://nacoesunidas.org/pos2015/agenda2030/, acessado em junho 2020

5. World Health Organization. The WHO End TB Strategy. Genebra, 2015, disponível em https://www.who.int/tb/post2015_strategy/en/, acessado em junho 2020 .

6. Zumla, A.; Memish, Z. A.; Maeurer, M.; Bates M.; Mwaba, P.; AlTawfiq, J. A.; Denning, D. W.; Hayden, F. G.; Hui, D. S.; Lancet Infect. Dis. 2014, 14, 1136.

7. Bloemberg, G. V.; Keller, P. M.; Stucki, D.; Stuckia, D.; Trauner, A.; Borrell, S.; Latshang, T.; Coscolla, M.; Rothe, T.; Hömke, R.; Ritter, C.; Feldmann, J.; Schulthess, B.; Gagneux, S.; Böttger, E. C.; N. Engl. J. Med. 2015, 373, 1986.

8. Kumar, P.; Capodagli, G. C.; Awasthi, D.; Shrestha, R.; Maharaja, K.; Sukheja, P.; Li, S.; Inoyama, D.; Zimmerman, M.; Liang, H. P. H.; Sarathy, J.; Mina, M.; Rasic, G.; Russo, R.; Perryman, A. L.; Richmann, T.; Gupta, A.; Singleton, E.; Verma, S.; Husain, S.; Soteropoulos, P.; Wang, Z.; Morris, R.; Porter, G.; Agnihotri, G.; Salgame, P.; Ekins, S.; Rhee, K. Y.; Connell, N.; Dartois, V.; Neiditch, M. B.; Freundlich, J. S.; Alland, D.; mBio 2018, 9, e02101-17.

9. Santos, M.; Massari, G.; Santos, D.; Fellows, L.; Parcerias Estratégicas 2004, 9,19

10. Pimenta, F. P.; TransInformação 2017, 29, 323.

11. Costa, E. C. S.; Freitas, J. J. R.; Freitas, J. C. R.; Quim. Nova 2018, 41, 713.

12. Santos-Gandelman, J.; Machado-Silva, A.; Mem. Inst. Oswaldo Cruz 2019, 114.

13. Niosi, J.; Technology Analysis \& Strategic Management 2016, 29, 251.

14. Barroso, W. B. G.; Quoniam, L.; Gregolin, J. A. R.; Faria, L. I. L.; World Pat. Inf. 2003, 25, 63

15. Vasconcellos, A. G.; Morel, C. M.; PLoS One 2012, 7.

16. Buainain, A. M.; Souza, R. F.; Propriedade intelectual, inovação $e$ desenvolvimento: desafios para o Brasil, ABPI: Rio de Janeiro, 2018.

17. Sinisterra, R. D.; Speziali, M. G.; Guimaraes, P. P. G.; Silva, A. M.; Quim. Nova 2013, 36, 1527.

18. Speziali, M. G.; Sinisterra, R. D.; Quim. Nova 2015, 38, 1132

19. Akkari, A. C. S.; Munhoz, I. P.; Tomioka J.; dos Santos, N. M. B. F.; dos Santos, R. F.; Gestão \& produção 2016, 23, 365.

20. Palmeira Filho, P. L.; Capanema, L. X. L. Em O BNDES em um Brasil em transição; Além, A. C., Giambiagi, F., eds.; BNDES: Rio de Janeiro, 2010, cap. 19.

21. Brasil, Lei $\mathrm{n}^{\circ} 13.243$, de 11 de janeiro de 2016. Dispõe sobre o estímulo ao desenvolvimento científico, à pesquisa, à capacitação científica e tecnológica e altera a Lei no 10.973, de 2 de dezembro de 2004, a Lei no 6.815 , de 19 de agosto de 1980, a Lei no 8.666, de 21 de junho de 1993, a Lei no 12.462, de 4 de agosto de 2011, a Lei no 8.745, de 9 de dezembro de 1993, a Lei no 8.958, de 20 de dezembro de 1994, a Lei no 8.010, de 29 de março de 1990, a Lei no 8.032, de 12 de abril de 1990, e a Lei no 12.772, de 28 de dezembro de 2012, nos termos da Emenda Constitucional no 85, de 26 de fevereiro de 2015. Diário Oficial da União, Brasília, 2016, disponível em http://www.planalto.gov.br/ ccivil_03/_ato2015-2018/2016/lei/113243.htm, acessado em junho 2020.

22. World Intellectual Property Organization. PCT - The International Patent System, Frequently Asked Questions, disponível em https://www. wipo.int/pct/en/faqs/faqs.html, acessado em junho 2020. 
23. Fonseca, B.P.F.; Fernandes, E.; Fonseca, M.V.A; Science and Public Policy 2017, 44, 37.

24. Pimentel, V. P.; Vieira, V. A. M.; Mitidieri, T. L.; Oliveira, F. F. S.; Pieroni, J. P.; Revista do BNDES 2015, 43, 41.

25. Thakral, S.; Singh, V.; Curr. Bioact. Compd. 2019, 15, 316.

26. Vitaku, E.; Smith, D. T.; Njardarson, J. T.; J. Med. Chem. 2014, 57, 10257.

27. Yerien, D. E.; Bonese, S.; Postigo, A.; Org. Biomol. Chem. 2016, 14, 8398.

28. Schlitzer, M.; ChemMedChem 2007, 2, 944.

29. Pissinate, K.; Rostirolla, D. C.; Pinheiro, L.M.; Suryadevara, P.; Yogeeswari, P.; Sriram, D.; Basso, L. A.; Machado, P.; Santos, D. S.; J. Braz. Chem. Soc. 2015, 26, 1357.

30. Stefanska, J.; Nowicka, G.; Struga, M.; Szulczyk, D.; Koziol, A. E.; Augustynowicz-Kopec, E.; Napiorkowska, A.; Bielenica, A.; Filipowski, W.; Filipowska, A.; Drzewiecka, A.; Giliberti, G.; Madeddu, S.; Boi, S.; La Colla, P.; Sanna, G.; Chem. Pharm. Bull. 2015, 63, 225.

31. Tatar, E.; Karakuş, S.; Küçükgüzel, S. G.; Okullu, S. Ö.; Ünübol, N.; Kocagöz, T.; De Clercq, E.; Andrei, G.; Snoeck, R.; Pannecouque, C.; Kalayci, S.; Şahin, F.; Sriram, D.; Yogeeswari, P.; Küu̧ükgüzel, I.; Biol. Pharm. Bull. 2016, 39, 502.

32. Lun, S.; Tasneen, R.; Chaira, T.; Stec, J.; Onajole, O. K.; Yang, T. J.; Cooper, C. B.; Mdluli, K.; Converse, P. J.; Nuermberger, E. L.; Raj, V. S.; Kozikowski, A.; Bishai, W. R.; Antimicrob. Agents Chemother. 2019, 63, e00343-19.

33. Marvadi, S. K.; Krishna, V.S.; Surineni, G.; Reshma, R. S.; Sridhar, B.; Sriram, D.; Kantevari, S.; Bioorg. Chem. 2020, 96, 103626.

34. Sharifi-Rad, J.; Salehi, B.; Stojanović-Radić, Z. Z.; Fokou, P. V. T.; Sharifi-Rad, M.; Mahady, G. B.; Sharifi-Rad, M.; Masjedi, M.R.;
Lawal, T.O.; Ayatollahi, S.A.; Masjedi, J.; Sharifi-Rad, R.; Setzer, W. N.; Sharifi-Radn, M.; Kobarfard, F.; Rahman, A.; Choudharyp, M. I.; Ata, A.; Iriti, M.; Biotechnol. Adv. (2017), doi:10.1016/j. biotechadv.2017.07.001.

35. Gautam, R.; Saklani, A.; Jachak, S. M.; J. Ethnopharmacol. 2007, 110, 200

36. Villanova, J. C. O.; Oréfice, R. L.; Cunha, A. S.; Polim.: Cienc. Tecnol. 2010, 20, 51 .

37. Xu, Z.; Meshcheryakov, V. A.; Poce, G.; Chng, S. S.; Proc. Natl. Acad. Sci. U. S. A. 2017, 114, 7993.

38. Takayama, K.; Wang, C.; Besra, G. S.; Clin. Microbiol. Rev. 2005, 18, 81.

39. Libardo, J. M.; Boshoff, H. I.; Barry, C. E.; Curr. Opin. Pharmacol. 2018, $42,81$.

40. Zhang, W.; Lun, S.; Wang, S. H.; Jiang, X. W.; Yang, F.; Tang, J.; Manson, A. L.; Earl A.M.; Gunosewoyo, H.; Bishai, W. R.; Yu, L. F.; J. Med. Chem. 2018, 61, 791 .

41. Grzegorzewicz, A. E.; Pham, H.; Gundi, V. A.; Scherman, M.S.; North, E.J.; Hess, T.; Jones, V.; Gruppo, V.; Born, S. E.; Korduláková, J.; Chavadi, S. S.; Morisseau, C.; Lenaerts, A. J.; Lee, R. E.; Mcneil, M. R.; Jackson, M.; Nat. Chem. Biol. 2012, 8, 334

42. Su, C. C.; Klenotic, P. A.; Bolla, J. R.; Purdy, G. E.; Robinson, C. V.; Yu, E. W.; Proc. Natl. Acad. Sci. U. S. A. 2019, 116, 11241.

43. Jeon, D.; Jeong, M. C.; Jnawali, H. N.; Kwak, C.; Ryoo, S.; Jung I. D.; Kim Y.; Molecules 2017, 22, 183.

44. O'kane, C. M.; Elkington, P. T.; Jones, M. D.; Caviedes, L.; Tovar, M.; Gilman, R. H.; Stamp, G.; Friedland, J. S.; Am. J. Respir. Cell Mol. Biol. 2010, 43,465 . 